\title{
Transposition
}

Musique et Sciences Sociales

$7 \mid 2018$

Le prix de la musique

\section{Aude Ameille, Pascal Lécroart, Timothée Picard \& Emmanuel Reibel (eds), Opéra et cinéma}

Rennes, Presses Universitaires de Rennes, 2017.

Jérôme Rossi

\section{CpenEdition}

\section{Journals}

Édition électronique

URL : http://journals.openedition.org/transposition/2381

DOI : 10.4000/transposition.2381

ISSN : 2110-6134

Éditeur

CRAL - Centre de recherche sur les arts et le langage

Référence électronique

Jérôme Rossi, « Aude Ameille, Pascal Lécroart, Timothée Picard \& Emmanuel Reibel (eds), Opéra et cinéma », Transposition [En ligne], 7| 2018, mis en ligne le 15 septembre 2018, consulté le 25 septembre 2020. URL : http://journals.openedition.org/transposition/2381 ; DOI : https://doi.org/ 10.4000/transposition.2381

Ce document a été généré automatiquement le 25 septembre 2020

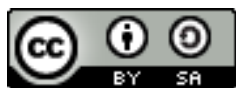

La revue Transposition est mise à disposition selon les termes de la Licence Creative Commons Attribution - Partage dans les Mêmes Conditions 4.0 International. 


\title{
Aude Ameille, Pascal Lécroart, Timothée Picard \& Emmanuel Reibel (eds), Opéra et cinéma
}

Rennes, Presses Universitaires de Rennes, 2017.

\author{
Jérôme Rossi
}

\section{RÉFÉRENCE}

Aude Ameille, Pascal Lécroart, Timothée Picard \& Emmanuel Reibel (eds), Opéra et cinéma, Rennes, Presses Universitaires de Rennes, 2017.

Dans le numéro spécial de l'Avant-scène opéra ( $\mathrm{n}^{\circ} 98,1987$, p. 6) consacré aux rapports entre opéra et cinéma, Ermanno Comuzio pouvait écrire :

Un vent de folie souffle sur l'opéra, tant à la scène qu'à l'écran. Plus qu'une mode, c'est un must, une obligation sociale, à tous les niveaux. Les hommes de spectacle font des pieds et des mains pour réaliser des mises en scène d'opéra; les chefs d'orchestre défendent des alliances jadis impensables avec des auteurs qui ne se limitent plus à faire évoluer des groupes sur scène ou à suggérer des mouvements aux chanteurs, mais proposent des 'interprétations globales'; les impresarios ne savent plus où donner de la tête; les cinéastes enfin, meurent d'impatience de porter les mélodrames à l'écran.

Plus de trente ans plus tard, l'enthousiasme autour des relations entre les deux arts n'est pas retombé et le livre Opéra et cinéma édité aux Presses Universitaires de Rennes en sonde les raisons avec minutie et érudition. Co-édité scientifiquement par quatre auteurs, émaillé d'entretiens (Philippe Béziat, Benoît Jacquot, Jacques Martineau, Michèle Reverdy et Olivier Simonnet), le recueil est articulé en cinq parties de tailles à peu près similaires. Il vient enrichir une bibliographie importante dans les pays anglosaxons, bien plus modeste en France. Dans un mouvement d'explication des origines, la première partie relate les premières manifestations de croisements entre les deux arts, tandis que les seconde et quatrième parties sont conçues en miroir: l'influence du 
cinéma sur l'opéra, puis de l'opéra sur le cinéma. Les troisième et cinquième parties sont respectivement consacrées à la captation et la diffusion de l'opéra (par la télévision, la vidéo et le cinéma), et aux «interactions multiples et multiformes » suscitées ou provoquées par les croisements entre les deux genres.

Dans leur introduction, après avoir montré comment les deux arts avaient pu avoir besoin l'un de l'autre - le cinéma a eu besoin de l'opéra pour se donner une légitimité artistique, l'opéra a eu besoin du cinéma pour offrir une alternative intéressante aux compositeurs alors qu'ils traversaient une crise profonde après la seconde guerre mondiale (à la fois esthétique, idéologique et morale, p. 14) -, les auteurs proposent une typologie de la présence opératique au cinéma :

- utilisation de l'opéra à titre de citation («La chevauchée des Walkyries» dans Apocalypse Now de Francis Ford Coppola, 1979);

- filmage d'une grande scène d'opéra intégrée à l'intrigue (Matchpoint de Woody Allen);

- intervention de personnages fictifs de chanteurs et de divas (Diva de Jean-Jacques Beineix, 1980), et biopics de chanteurs d'opéras (Callas Forever, Franco Zeffirelli, 2002);

- film-opéra, genre hybride au sein duquel les auteurs distinguent l'opéra en prose (reprise de la trame d'un opéra dans un film entièrement parlé), le film paraphrase (qui suit l'intrigue de l'opéra source de près, et lui adjoint ses airs les plus célèbres) et le film-opéra proprement dit, qui suit de près la partition de l'œuvre source (donc entièrement chanté).

4 En retour, l'influence du cinéma est bien présente à l'opéra dans les sujets (Romanza de Sergio Rendine, par exemple, tire son origine des Ailes du désir de Wim Wenders, 2002), la confection des livrets (les compositeurs parlent plus volontiers de « séquence » que de «scène»), la mise en scène qui peut inclure un dispositif de projection cinématographique ; enfin, la captation audiovisuelle de l'opéra permet à celui-ci une retransmission télévisée, une diffusion dans les salles de cinéma (en temps réel) ou une distribution en dvd.

5 La première partie s'ouvre par la présentation de deux inventeurs, William K. L. Dickinson et Louis A.A. Le Prince qui associent l'opéra à leurs premières expériences cinématographiques. On peut regretter la brièveté de l'article et le déséquilibre en faveur de Dickinson; le texte a toutefois l'intérêt de montrer combien les débuts du cinéma recèlent encore de fascinantes découvertes. Deux autres articles sont consacrés aux œuvres du cinéma muet. Le premier présente le travail d'adaptation et - ce que l'on sait moins - de composition du chef d'orchestre du cinéma Gaumont-Palace, Paul Fosse ; ce dernier réservait les morceaux extraits d'opéras - la plupart du temps en y soustrayant la partie vocale - aux œuvres aux forts enjeux dramatiques. Le second texte retrace la généalogie du fantastique dans le cinéma américain et pose l'adaptation de The Phantom of the Opera (réal. R. Julian, 1925) comme l'une des œuvres clés à cet égard dans ses rapports au corps, à la danse et à la musique. Mis en avant par Méliès dans ses applications cinématographiques, les trucages ne bénéficient pas de la même valorisation à l'opéra ; Marie-Anne Le Roy Maršálek nous révèle ainsi que la critique de la Belle Epoque préfère se concentrer sur le rendu mystique des adaptations lyriques de Jeanne d'Arc plutôt que sur l'aspect spectaculaire des mises-en-scène, bien que celui-ci soit un atout commercial de premier plan. Enfin, les articles de Van der Hoeven et Plasseraud étudient la postérité wagnérienne de l'idée de synthèse des arts à travers respectivement l'idéalo-wagnérisme, courant parisiano-bruxellois qui se situe à la croisée du symbolisme et de la synesthésie avec pour principaux représentants Joseph Péladan (le fameux Sâr Péladan pour lequel Erik Satie composa Les sonneries de la 
Rose+Croix et L'Air du Grand Maitre), Albert Cozanet (pseudonyme de Jean d'Udine), Alexandre Scriabine et Edgard Varèse, et l'unanimisme, qui est d'ailleurs moins un courant que la recherche constante de réactualisation de la tragédie grecque - en tant qu'elle représente un monde dans lequel l'art est le garant d'une fusion spirituelle des individus, fusion dont le drame wagnérien avait été la réalisation la plus aboutie et que le cinéma se promettait de devenir.

6 La confrontation de l'opéra au film implique de repenser le premier, tant sur les plans esthétique que sociologique, car l'œil humain s'est habitué à une nouveau type de représentation de la réalité (pour A. Schoenberg : « le film a gâché l'œil du spectateur », p. 91). Premier auteur de cette seconde partie, Julien Segol nous montre comment l'opéra, genre conservateur par excellence, a su s'adapter en Allemagne en prenant le cinéma comme " horizon d'attente ». La résurgence de la pantomime, l'expressivité des regards, le recours aux "encarts» (équivalents des sous-titres), la rapidité des changements de scènes sur le modèle du montage cinématographique, ou encore les projections de films sur scène (jeux de miroirs qui défamiliarisent la présence du corps sur scène) constituent autant de traits empruntés au cinéma muet de l'époque, qui apparaît comme une nouvelle "poétique du spectaculaire» (p. 96) sur laquelle se refonde la représentation opératique germanique, faisant preuve d'une admirable adaptabilité. Les autres articles de cette partie s'attachent tous à montrer comment le cinéma a concrètement pénétré le monde de l'opéra : cela se traduit, chez Eisenstein, par une utilisation extrêmement précise des éclairages au service d'une expressivité plastique pour sa mise en scène de La Walkyrie au Bolchoï, tandis que l'on peut parler d'une véritable "conception cinématographique» de l'opéra chez Peter Eötvos: simultanéité d'action inspirée du split screen (trois scènes dédoublées), rôle signifiant de la musique avec l'attribution de notes de références aux personnages ou encore soin accordé au bruitage. Les tableaux détaillés d'Aude Ameille (p. 122-132) montrent l'importance, depuis le début des années cinquante, d'un phénomène consistant à confier la mise en scène d'un opéra à un cinéaste. Du point de vue de l'opéra, cette stratégie peut se comprendre comme une volonté de toucher un public plus large que celui des «habitués ", mais les enjeux vont bien au-delà. Si les difficultés sont réelles pour le cinéaste (jeu théâtral des chanteurs, absence de gros plans), l'opéra lui donne en retour la possibilité de se laisser guider par la musique : « ce n'est pas le livret qui constitue le 'scénario du spectacle' mais bien la musique " (Benoît Jacquot, p. 136). L'auteur termine son propos en se posant l'intéressante question d'une esthétique de la mise en scène qui serait propre aux réalisateurs.

7 Delphine Vincent débute la troisième partie de l'ouvrage en examinant les stratégies des cinéastes lorsqu'ils veulent transposer un opéra au cinéma. Si la réduction temporelle de l'opéra vers le cinéma est quasiment inévitable - pour correspondre à une durée moyenne d'une heure trente -, les solutions retenues sont, elles, très diverses et ne se laissent pas classer en deux catégorie comme l'historiographie courante nous le laisse penser (rupture au début des années soixante-dix): coupures, ajout de séquences ou de voix-off, traduction, chant "en direct» ou en playback, actualisation ou non dans notre société, doublage ou non des chanteurs par des acteurs, manipulations sonores en fonction des plans... les appropriations sont multiples et riches. On louera la vingtaine de pages d'annexes de ce seul article qui recense toutes les modifications pratiquées par les processus d'adaptation des opéras cités. «Synthèse du hasard et de la nécessité » (p. 176), le film-opéra de Danièle Huillet et Jean-Marie Straub sur Moses und Aron de Schoenberg relève - contre le doublage et les 
manipulations de post-production - le défi conjoint du tournage en plein air et du chant en direct, entre le sonore imprévisible et la musique en tant qu'œuvre d'art programmée par l'artiste ; remarquons le dispositif, assez insolite, consistant à avoir enregistré tout l'opéra dans une version purement instrumentale, afin que les chanteurs puissent ensuite chanter en direct pendant le tournage. Concernant les rapports entre le sonore et le visuel, ceux-ci «sont comme deux jets qui s'affranchissent et gagnent en autonomie", en une ambivalence dynamique qui cherche à " capter les conditions qui ont engendré cet opéra, puisque Schoenberg, au début des années 1930, est celui qui a su retranscrire le cri qui parcourt le monde en musique» (p. 180). Les quatre autres articles de cette partie se concentrent sur la présence de l'opéra à la télévision en l'abordant sous divers angles: l'histoire des premières mises en scène spécialement conçues pour la télévision et les captations (Marie Auburtin), le dvd et son livret (David Christoffel), le sous-genre du documentaire sur l'opéra (Elizabeth Giuliani, François-Gildas Tual). Cette place accordée au medium télévisuel est rare en musicologie et atteste de la richesse inouïe de ce media pour l'étude de l'évolution des genres musicaux et des mentalités au $\mathrm{xx}^{\mathrm{e}}$ siècle. Pour chacune de leur proposition, les auteurs proposent des typologies efficaces et pertinente. Le tableau de F.-G. Tual qui classe le matériau cinématographique en le répartissant sur un continuum allant de la forme la plus concrète (réalité quotidienne : paysage) à la plus abstraite (le carton silencieux) nous a semblé particulièrement perspicace.

8 La quatrième partie traque la question de l'« opératique » au cinéma - une notion qui sera articulée avec celle de "cinématographique» dans le tout dernier article du volume - dans les figures de la diva (la chanteuse, l'actrice, le personnage de fiction) au cinéma (Thierry Santurenne), les adaptations du roman de Gaston Leroux « Le fantôme de l'opéra » (Timothée Picard), le cinéma de Tsui Hark (Simon Daniellou), les films de Dario Argento (Pierre Jailloux), Match Point de Woody Allen (Justin Bernard) ; parmi les éléments avancés, on retient l'affirmation d'une rhétorique de la mise en scène (par exemple dans le caractère ostentatoire des entrées et sorties des musiques), une certaine manière d'occuper l'espace avec des mouvements de caméra en trois dimensions et, de manière plus générale un goût pour l'outrancier associant « décorum, performance, paroxysme, lyrisme, stylisation, artifice, totalité, escapisme, kitsch » (Picard, p. 261). Pour Justin Bernard, Match Point (réal. Woody Allen, 2005) présente l'opéra à la fois comme marqueur social, fil conducteur du drame et expression d'un drame interne. Dans la recherche d'une filiation entre l'opéra et le cinéma, Laurent Guido questionne la vision rétrospective de Wagner comme celle d'un génie visionnaire, père spirituel du spectacle cinématographique. Si la configuration particulière du théatre de Bayreuth est bien une véritable salle de cinéma avant l'heure, les aspects les plus spectaculaire de l'opéra wagnérien - actions virevoltantes, effets lumineux (l'auteur cite la lanterne d'Eugène Frey pour la chevauchée des Walkyries, l'éclairage électrique pour Parsifal), importants dispositifs scéniques (le panorama mobile) - sont moins destinés à impressionner le public - l'article montre que les trucs et effets de machinerie sont courants à l'époque - qu'à tenter de mettre en œuvre le Gesamkunstwerk pour lesquels ils s'avèrent "constituer des éléments minimaux nécessaires à l'expression» (p. 325). Dans un article dense, João Pedro Cachopo expose les idées de Stanley Cavell, philosophe américain, pour lequel la relation conflictuelle entre opéra et cinéma contribue à définir leur identité ; on pourra toutefois regretter la concision de l'article qui, selon nous, peine à rendre justice à la complexité de la pensée de Stanley Cavell. Enfin, le texte ambitieux de Patrick Longuet 
(d'après Bazin) pense le cinéma et ses rapports à l'opéra à travers l'idée d'impureté, distinguant similitudes externes (univers de vedettes, industrie culturelle) et «relations internes plus profondes » qu'il explore dans neuf films qui intègrent divers aspects de la création opératique (faste du décorum, effet de rideau, scènes chantées, prologues, sons en coulisse) ; pour l'auteur, la différence principale entre les deux arts l'opéra (comme le théâtre) est fondé sur la coprésence, dans le même espace, des acteurs et des spectateurs - peut être dépassée en intégrant un public fictionnel à l'image cinématographique.

La dernière partie, plus disparate, reste néanmoins passionnante car riche en découvertes et en propositions. Béatrice Gandois étudie le travail d'arrangement de Korngold sur la partition de Mendelssohn dans le film A Midsummer Night's Dream (réal. M. Reinhardt and W. Dieterle, 1935), superproduction hollywoodienne qui s'approche du mélodrame par la quantité de musique présente ; une comparaison avec le récent film éponyme de M. Hoffman (1999), qui intègre des extraits d'opéras italiens, aurait offert un éclairage contemporain intéressant. Cécile Carayol montre l'influence de Debussy sur le cinéma hollywoodien, peut-être moins perceptible mais toute aussi prégnante que celle de Wagner. Avec une scène de Rebecca (réal. A. Hichcock, comp. F. Waxman, 1940), elle montre notamment comment le musicien français, avec Pelléas et Mélisande et sa prosodie proche de la parole parlée, a posé les bases de la technique d' underscoring, une écriture musicale spécifiquement cinématographique consistant à épouser les contours du langage parlé pour offrir un écrin musical empathique aux dialogues. " Oratorio dramatique ", " mimodrame ", « mystère lyrique ", Jeanne d'Arc au bûcher est une œuvre qui témoigne de l'influence croisée de l'opéra et du cinéma dans le but de renouveler le genre lyrique ; Pascal Lecroart raconte quelques étapes cruciales dans la création et la réception de cette œuvre, vraisemblablement influencée par un film (le Faust de F. W. Murnau, 1926), mise en musique par Arthur Honegger, compositeur rompu aux exigences de la musique de film (il en a produit six dans les trois années qui précèdent), puis mise en scène et adaptée à l'écran par Roberto Rossellini (1954). Opéra de M. Beretti et d'A. Bon conçu pour le cinéma, La Jeune Fille au livre (réal. J-L. Comolli, 1994) revisite les rapports traditionnels entre les divers arts de la littérature, de la musique, de la peinture - un tableau constitue le centre de l'intrigue - et du cinéma; pour Elisabeth Rallo Ditche, ce dernier fonctionne comme un "opérateur de lecture", c'est-à-dire qu'il sélectionne un sens privilégié pour le (télé)spectateur, ici la question des rapports entre l'art et la réalité. Expérience inédite, Atiq Rahimi a tiré du récit Terre et cendre - à forte résonance autobiographique - un livre, un film et un opéra ; Xavier Rockenstrocly en analyse les diverses adaptations et transformations permettant d'approfondir les spécificités expressives des trois médias. Dernière étude de cas, The Tristan Project propose une réflexion sur l'apport de la vidéo - le film de Bill Viola - à la mise en scène de Peter Sellars pour la version de Tristan und Isolde proposée à l'Opéra Bastille en 2005 ; complément spirituel, la partie vidéo couvre l'ensemble de la représentation, imposant l'eau comme élément symbolique central voir l'ascension finale de Tristan transposée dans le monde sous-marin - et manipulant la temporalité par les effets de lenteur, de ralenti ou de slow motion. Si un long et passionnant entretien fait office de conclusion, l'article d'Emmanuel Reibel et Céline Carenco qui questionne les notions de "cinématographique» à l'opéra et d' "opératique » au cinéma dans les critiques d'œuvres offre une magistrale réflexion finale sur la question, insistant sur la dissymétrie entre les deux arts: le cinéma représente une forme de culture universellement partagée, porteuse ou non de valeurs 
artistiques, tandis que l'opéra constitue "le modèle d'un rapport au monde qui esthétise la violence, embrasse une forme de totalité, entretient un rapport distendu à la temporalité, et hisse en fin de compte le propos à un niveau transcendant le réel. » (p. 435)

Si les rapports entre opéra et cinéma ne cessent de fasciner, c'est que les deux arts continuent de poser la question fondamentale du naturalisme et du rapport au réel. Les entrées multiples du livre (le wagnérisme, les trucages, les techniques d'adaptation, les genres filmiques, la mise en scène) permettent d'en étudier les multiples facettes, faisant assurément de ce collectif brillamment mené un ouvrage de référence.

\section{AUTEURS}

\section{JÉRÔME ROSSI}

Maître de conférences en musicologie à l'université de Nantes, Jérôme Rossi est l'auteur de nombreux ouvrages et articles consacrés à la musique post-romantique - particulièrement la musique anglaise de la première moitié $\mathrm{du} \mathrm{xx}^{\mathrm{e}}$ siècle - et aux liens qui unissent musique et cinéma. Son livre sur l'œuvre du compositeur anglais Frederick Delius a obtenu le Prix des Muses de la biographie en 2011 ; il a récemment publié l'ouvrage La musique de film en France chez Symétrie (Lyon). Il est le compositeur de nombreuses musiques de documentaires et de courtsmétrages. 